\title{
Blockchain-based Electronic Data Storage and Certificate System of Housing Provident fund
}

\author{
Yang Song ${ }^{1}$, Yue $\mathrm{Gu}^{2}$, Xue Chen ${ }^{1}$, Xiaojun $\mathrm{Zhu}^{3}$ and Keting $\mathrm{Yin}^{3+}$ \\ ${ }^{1}$ Chongqing Municipal Housing Provident Fund Administration Center, China \\ ${ }^{2}$ Hangzhou Echaincity Technology Co., Ltd., China \\ ${ }^{3}$ School of Software Technology, Zhejiang University, China
}

\begin{abstract}
At present, China is gradually improving the housing provident fund related businesses, however, the existing centralized system still cannot meet the needs of online business management, personal privacy protection, electronic evidence effectiveness and so on. To solve these problems, this paper proposes a blockchain-based electronic storage and certificate system of housing provident fund. With the establishment of blockchain-based data sharing platform, it supports Chongqing Housing Provident Fund Management Center to form evidence consensus mechanism. Through the construction of the "hands-on" witness facility system of the judicial institutions, data collection, trusted storage and management of the co-investigation data of provident fund business and key business processes are carried out, so as to ensure the validity, authenticity and legal effect of the certification materials.
\end{abstract}

Keywords: housing provident fund, blockchain, storage certificate

\section{Introduction}

Housing provident fund is a long-term housing fund enforced by the state and plays an important role in China's urban housing security system [1]. Housing provident fund, which has the specific characteristics and attributes, guarantee and mutual aid, gathers limited individual capital to form stable, sustainable capital source, and helps workers solve housing problems by issuing provident fund loans and withdrawing funds to pay for the house purchase. Blockchain technology has been applied to construct the national housing provident fund data platform, docking with the national government affairs service platform and the State Council client.

Under the influence of the factors, such as the surge of online business, complex information interaction brought by different service channels, information synergy needs across regions and departments and so on, it is difficult for traditional centralized business management system to provide effective self-certification for the provident fund center. By using blockchain to build the electronic storage and certificate system of housing provident fund, this paper aims to solve the current problems encountered in Chongqing housing provident fund business management. Overall, the main contributions of this paper can be summarized as follows:

-Firstly, we apply blockchain into the provident fund business to realize the deposit of the investigation data and the whole process of the key business, so that the proof materials can be traced completely and the provident fund business is handled conveniently and reliably.

-Secondly, the files are stored in the internal nodes of the provident fund center, and generate the unique data fingerprint and upload to blockchain. The system not only introduces external nodes to participate in the consensus mechanism, but also realizes data encryption and privacy protection.

-Thirdly, we have set up the Intelligent Customer Service Consortium Blockchain cooperating with China Merchants Bank, and stored the voice interaction data related to the intelligent customer service platform to guarantee the authenticity and reliability of the data, and conduct effective supervision and audit.

+ Corresponding author. Tel.: +86-0574-27830788; fax: +86-0574-27830606

E-mail address: yinkt@zju.edu.cn 
The rest of paper is organized as follows. In Sect. 2, we discuss the problems and demands of Chongqing provident fund business management at the present stage, and expounds the advantages of blockchain application in the provident fund field. In Sect. 3, related works on the application of blockchain in the field of storage and certificate of provident fund are introduced. In Sect. 4, we provide an overview of system design, covering business process and architecture. In Sect. 5, the special program of the system is presented. We discuss the complete procedures, including authentication, internal data acquisition, external data acquisition, data storage, data backup and archiving. In Sect. 6, the practicability of the system is proved through case analysis. Finally, we conclude with the discussion and future work in Sect. 7.

\section{Motivation}

At present, the main business data generated in the process of handling Chongqing housing provident fund business are managed by the center itself. It is hard for the traditional centralized business system to prove its innocence in the case of business disputes or users' doubts, and it usually requires a third party with public credibility to help prove that the business data has not been falsified. Simultaneously, because some dynamic assistance information provided by the external units need to be obtained in the process of special business of the provident fund, the timeliness and correctness of these information may be questioned. Therefore, we need to store the external information obtained at the time of business processing.

The blockchain established by the provident fund center and external units can save important files and data on the chain immediately, and upload evidences to the blockchain with a timestamp to make sure that the data cannot be falsified. Important evidence can be provided when traceability is required, so as to ensure the credibility of the stored data. Moreover, the blockchain-based evidence platform can help us review the time, place, main equipment and other elements of the occurrence of the links, such as the generation, collection, transmission, storage, verification of the electronic data comprehensively. That is to say, each communication data of key business is stored independently to provide reliable evidence-based guarantee for each business.

\section{Related Works}

In recent years, domestic and foreign researchers have carried out a great deal of researches on the application of blockchain technology in many fields. X. Li [3] believes that blockchain has great application potential in the field of storage certificate, and he puts forward a credible electronic bidding system based on blockchain, in order to address issues, including operational compliance, multilateral coordination, and network security, which exist in distribution, validation, and backtracking of bidding data files. The system links different stakeholders together, with the help of the Consortium Blockchain, to form a flat trust management model. A. Buzachis[4] et al. describe a "blockchain-as-a-service" health information exchange system (BAAS-HIE) that uses private blockchain and smart contracts as access control managers for medical records. All health data is encrypted and stored in a decentralized Internet Protocol File System (IPFS), and hashes of asset URIs are stored in the blockchain. This solution is feasible in providing decentralized and fine-grained accessibility mechanisms for patients and physicians in a given health-care system. In order to solve risks and inconveniences of paper contracts, Qing Zhang[5] et al. propose a Blockchain-based contracts signing and data sharing prototype named FutureText using Hyperchain to sign Privately Offered Fund (POF) contracts, which helps to share privacy information among stakeholders while keeping forged signature, counterfeited seal and twin-contracts away. Besides, the encrypted contracts are put on the chain and original contracts are kept on local database using heterogeneous storage.

Nowadays, many scholars have made many studies about the innovative management of the provident fund system. Zhang Jing [6] analyzes the current situation of the management of housing provident fund in the era of big data and the imbalance between social demands and information, and points out that big data technology should be fully utilized to promote the innovation of the management of housing provident fund, in order to ensure that housing provident fund plays a greater role in social and economic development. $\mathrm{Li}$ Fei [7] explains the importance of legalization and utility of provident fund archives management, the advantages and disadvantages of provident fund electronic archives and the differences between electronic 
and paper archives. He also proposes several methods to solve the risk problems of provident fund electronic archives, fundamentally promoting the healthy and stable development of the management.

According to the survey, there are not many blockchain-based provident fund systems at home and abroad. Ant Technology has worked with CEC to create a "joint deposit certificate and trust-breaking punishment cloud platform"[8] to help realize the cross-center and cross-regional sharing of provident fund certificate and blacklist. The platform uses blockchain to share data, and all the urban housing provident fund management centers just need to access the platform and upload the blacklist to the blockchain, so that they can realize trusted record sharing, tamper-proof, non-denial and traceability. At the same time, the platform has the technical interconnection of government regulatory departments.

On the basis of the existing work, in order to create a better provident fund system, we apply blockchain into the system to solve the inconvenience of data sharing and the whole process of business evidences in the existing system.

\section{System Design and Implementation}

\subsection{Business Process}

Fig. 1 shows the business process of the electronic data storage and certificate system of the provident fund.

Blockchain guarantees the security and reliability of system data. According to the demand analysis of the existing system, internal data collection is performed by embedding automatic data collection points in key links or manually submitting certificates for storage, while external data collection is completed under the joint witness of other nodes of the consortium chain. Then the collected data is converted through solid certificates, and relevant evidences are saved and uploaded to the chain through the consensus platform and smart contract. The system offers auxiliary support for the provident fund business system by providing storage, backup, archiving and statistics of the data. In case of business disputes or user queries, the corresponding withdrawal and verification of the evidence can be carried out on and off the chain.

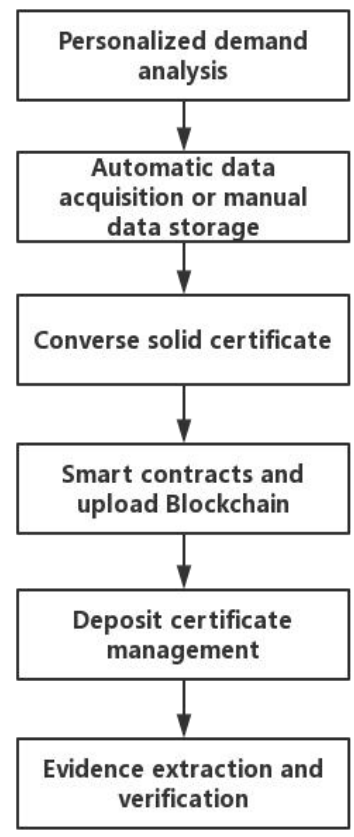

Fig. 1: Business procedure of provident fund electronic data storage and certificate system

\subsection{System Architecture}

The system structure of the electronic storage and certificate system of housing provident fund is divided into four layers, as shown in Fig. 2.

(1) First Layer: Data Storage Layer 
The underlying data should support storage technologies such as on-chain and off-chain storage, distributed storage networks, and relational storage systems, as well as storage expansion, data archiving, cold storage backup to ensure the safe and credible storage of electronic evidence and guarantee that the read and write performance will not be affected when the amount of system data increases continuously.

\section{(2) Second Layer: Basic Service Layer}

The basic service layer is the foundation, which can provide the underlying blockchain basic platform environment, including consensus, network, storage, and smart contract, and support blockchain node management and block data maintenance. The layer also can realize the writing of business logic in the blockchain and monitor system performance, data access and exchange and other information to ensure the stable operation of the blockchain system. We choose Hyperchain[9] blockchain underlying platform and the high-efficiency Byzantine consensus algorithm NoxBFT, which can improve the overall transaction throughput and system stability. The platform supports multiple smart contract programming languages such as Solidity and Java, and provides perfect contract life cycle management, which has features of programming friendliness, contract security and efficient execution, so as to adapt to changing and complex business scenarios. The pluggable encryption mechanism is adopted to encrypt the data, users, communication connection and other aspects involved in the whole life cycle of the business with different strategies.

\section{(3) Third Layer: Core Business Layer}

The business layer mainly includes general service, account service, forensic service, deposit management, data visualization and back-end management, etc. General services mainly include account and node authority control, blockchain data archiving, version recording and upgrading, and data partitioning. Account service mainly controls the authority of each account in the contract and provides basic user verification functions. The data collection sources of forensic services are divided into internal data and external data of the business system. Deposit management provides auxiliary support for provident fund business, mainly including query, verification, download and export of evidence. Data visualization supports monitoring of blockchain nodes, blocks, transaction data and contract status, and makes relatively intuitive statistics and display, which is convenient for operation and maintenance administrators to understand and analyze the operation of blockchain nodes in real time. The back-end management mainly monitors and warns the transactions and contracts of the storage and certificate system.

\section{(4) Fourth Layer: Access Layer}

The access layer provides the access for the storage certificate system, establishes a connection with the provident fund business system and the external co-check data platform, and connects with the lower-layer services through the API, and offers access to the external system.

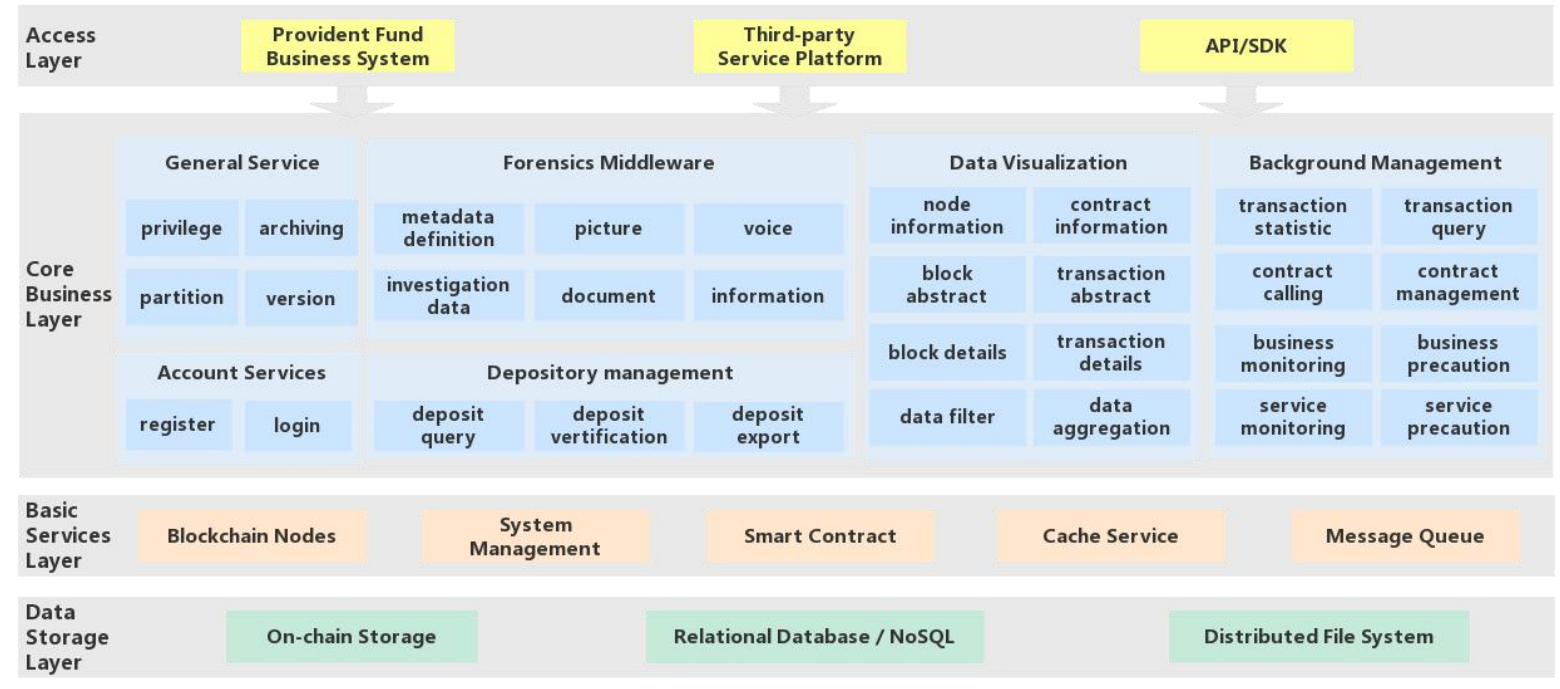

Fig. 2: System Architecture: four layers 


\section{The Specific Program}

This section mainly introduces the specific implementation of the blockchain business in the business layer of the system architecture, as shown in 4.2, including internal data collection of the business system, external data collection of the business system, data storage, data backup and archiving.

\subsection{Internal Data Acquisition}

The internal data collection of the business system mainly includes electronic application forms, electronic receipts and scanned pictures of paper materials submitted by users in offline business processing that can be generated from business data during the process of key provident fund business processing. By monitoring the related tables of the underlying relational database of the business system, newly generated data is discovered with database trigger technology or periodic polling and comparison, an then the system encapsulates and stores the data according to the relevant format, and automatically generates the corresponding metadata information and saves it on the chain. In a word, the internal data of the business system is directly generated by the business system at the time of the specific business to complete the data collection by the intuitive and visual evidence file, and then the consortium blockchain provides a credible timestamp for data storage.

\subsection{External Data Acquisition}

The external data collection of the business system mainly uses forensic middleware, which is mainly for active external data acquisition. The data forensics process is completed under the common witness of credible third-party nodes such as external judicial institutions in the consortium blockchain. In this process, the forensic middleware establishes an independent boundary between the internal business system and the external data service, and transfers the data sent by both parties in real time. It can prove the time, data and network behavior of the communication between the two parties in the blockchain-based method based on evidence, and generate electronic evidence certification documents through a visual interface to provide realtime and comprehensive evidence preservation for each key business involving external data acquisition. Meanwhile, based on the blockchain consensus capability, the verification information of external data is synchronized to the witness organization in case of future data disputes.

There are two situations for actively obtaining external data through forensic middleware, one is that the business system directly calls the external interface, and the other is that personnel business accesses the external business system to query the result screen-shots of evidence.

The direct call of the business system to the external interface is mainly established through the HTTP(s) protocol, and the request is forwarded to the forensic middleware through the setting of the relevant gateway. Then the forensic middleware combines the network layer TLS key exchange, the protocol layer head data, and the application layer electronic signature to realize network interaction and data verification, and forward the request results to the business system. In the process, the forensic middleware generates electronic evidence package files and metadata definition information in real time from the initiator IP information, receiver IP information, request URL, port, request message, and external port response message, and stores the data synchronously in real time through the blockchain, to ensure the consistency of the time of data occurrence and network interaction behavior information.

In the other way, the business personnel access the external business system under the built-in browser of the forensic middleware, and after obtaining the query results, call the forensic middleware screenshot program to take a screenshot of the content of a given URL. And the forensic middleware automatically obtains the resource information, network routing, server information, certificate information and other data of the target URL, generates electronic evidence package files and metadata definition information, synchronizes the data through the blockchain in real time, and finally returns the corresponding screenshots to business staff for follow-up business operations.

\subsection{Data Storage}

According to the type of storage data, the storage data can be divided into image type, voice type, document type, and information type (external data interaction process, SMS interface call process, etc.). 
Among them, the image category mainly includes face recognition live photos, screenshots of website query results, and scanned paper materials, etc. The voice category is mainly recording files of intelligent voice customer service and user interaction processes. The document category mainly consist of electronic application documents generated by customer application information in the business system and electronic receipt documents generated by business processing results. The information category is mainly electronic evidence files generated by a visual template to prove the time, data and network behavior of the communication between the two parties based on the blockchain.

Data storage needs to solidify the electronic evidence for storage. The corresponding file hash is obtained by using the hash calculation for the electronic evidence file, which is convenient for the on-chain and off-chain consistency comparison. The credible timestamp can be applied to electronic evidence documents by correlating credible time sources. The trusted timestamp service within the consortium blockchain can be established based on the smart contract and oracle technology, and the consortium blockchain timestamp service can be written through the smart contract. When the metadata of the electronic evidence file is written into the blockchain, the hash value of the electronic evidence file is signed by the private key related to the identity of the blockchain. For the electronic data obtained through the external query, a request to upload to the chain is initiated in real time to avoid the possibility of malicious tampering in the future.

The storage structure diagram of the blockchain is shown in Fig. 3.

According to the storage situation, the evidence data is divided into on-chain storage and off-chain storage. The on-chain storage adopts distributed storage to store the metadata definition information of the electronic evidence file, including the hash value of the electronic evidence file, trusted timestamp, related business serial number, storage node records, etc. The immutability of the blockchain guarantees that the metadata information of the electronic evidence file cannot be tampered with, and the smart contract provides the ability to detect metadata information. The off-chain storage adopts the distributed method based on DHT to store electronic evidence files and other contents. In order to protect the security of the certificate storage, it needs to designate nodes for storage. Each node is responsible for a small range of routing and stores a small part of data at the same time, so as to realize the addressing and storage of the DHT network [10].

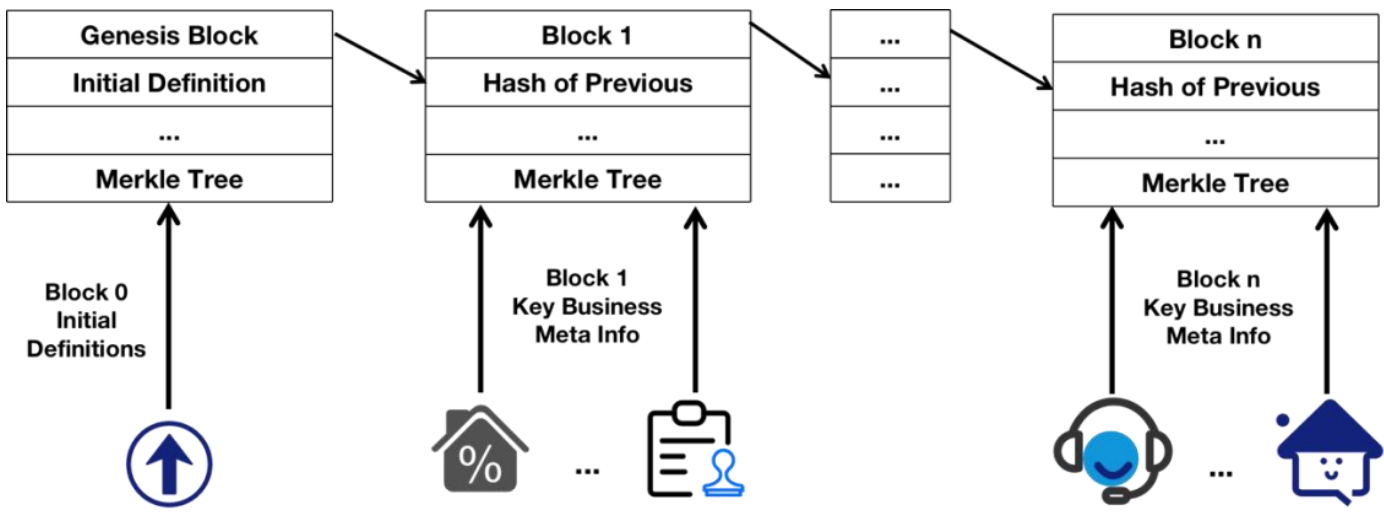

Fig. 3: Blockchain and Block Structure: The block body stores the parameters, timestamp and meta information.

\section{Case Study}

This section mainly demonstrates the usability and availability and reliability of this system through an application case of the intelligent customer service voice interaction certificate.

Chongqing Housing Provident Fund Management Center cooperated with China Merchants Bank and China Telecom to establish an "intelligent customer service platform", which is based on artificial intelligence technology, providing $7 * 24$ hours of online voice services to meet various inbound and outbound business scenarios. The following is a specific application. We use intelligent customer service's non-consulting voice call and interaction design, which includes overdue reminders and repayment reminders, to clearly record the relevant information that the recipient has learned in the voice file, and use the blockchain platform to store evidence of voice interaction and other data related to the intelligent 
customer service platform. Then Telecom provides a third-party certification of the actual call record and the voice files hash to form an effective legal evidence. In the future, it will be possible to further develop key business acceptance voice recording services based on intelligent customer service. This practical application guarantees the authenticity of the voice interaction data, and also effectively supervises and evaluates the platform based on the voice interaction data.

The process of the three-party node certificating the voice interaction data is shown in Fig. 4. China Telecom provides the intelligent customer service voice interactive network channel. When the call is over, China Telecom node will record the call time, call number, and call duration on the chain in real time. China Merchants Bank is responsible for providing intelligent customer service. After the call, the China Merchants Bank node saves the call recording file and the voice-to-text file generated in real time off the chain, and the hash value of the call recording file is stored on the chain in real time. Chongqing Housing Provident Fund Management Center is in charge of voice verification and certificate backup. When the network is idle, the CMB node will synchronize the call recording file and the voice-to-text file to Chongqing Housing Provident Fund Management Center node for backup storage. The Chongqing Housing Provident Fund Management Center node will verify whether the recording file corresponds to the recording file hash value on the chain, and checks whether the recording duration and the call duration recorded by China Telecom are within a reasonable error range. As a result, the three parties reach a consensus and make it clear that the call recording record has not been modified and is true.

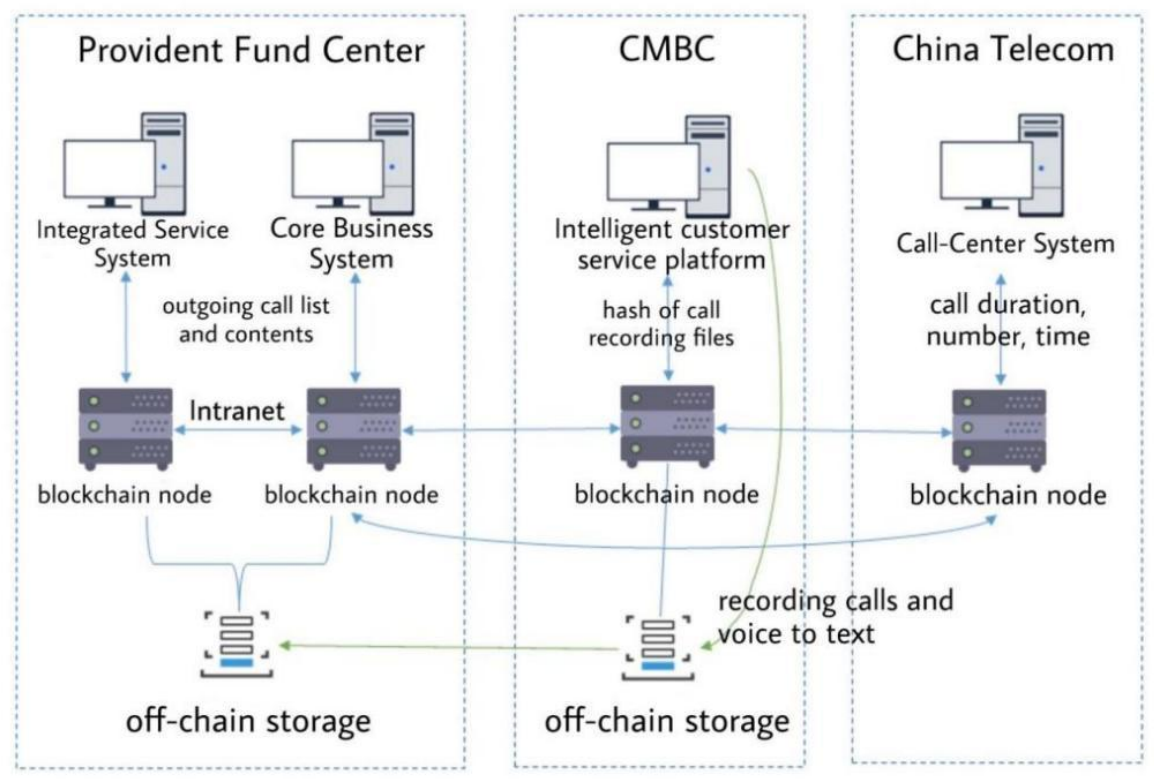

Fig. 4: Voice interactive data storage

\section{Conclusion and Future Work}

This paper proposes to construct a credible housing provident fund electronic data storage and certificate system based on blockchain to collect, store, and manage shared co-check data and key business processes, and realize four goals which include the traceability of the evidence and the whole process of key business, the isolation between storage and certificate, and the protection of the privacy of business data. At present, Chongqing Provident Fund Center is cooperating with China Merchants Bank to establish an intelligent customer service system to handle provident fund consultation and business processing through voice recognition, automatic response and artificial intelligence, and utilize blockchain to record evidence.

In the future, the project hopes to obtain tax and social security data to screen high-quality companies to expand the provident fund service, and proposes a sharing plan that "data will not be released, only model calculation results will be returned". At the same time, the investigation found that the provident fund business systems of various cities in Sichuan Province currently have a large gap, and blockchain can be considered for interactive collaboration. 
In order to maximize the advantages of the blockchain and strive to solve the above problems, we will continue to conduct research and improvement to provide further technical support for the housing provident fund storage and certificate system.

\section{Acknowledgment.}

This research was supported by the National Key R\&D Program of China No. 2019 YFB1404903.

\section{References}

[1] Wu Yidong, Chen Jie. Security or mutual assistance: A study on the housing provident fund system in China [J]. Chinese Journal of Administrative Management,2020(9):58-66.

[2] Ministry of Housing and Urban-Rural Development, Ministry of Finance, People's Bank of China. National housing provident fund 2019 annual report [N]. China Construction News,2020-06-15.

[3] X. Li, BCES: A BlockChain based Credible E-Bidding System, 2020 IEEE 6th International Conference on Computer and Communications (ICCC), Chengdu, China, 2020, pp. 2415-2421, doi: 10.1109/ICCC51575.2020.9345176.

[4] A. Buzachis, A. Celesti, M. Fazio and M. Villari, On the Design of a Blockchain-as-a-Service-Based Health Information Exchange (BaaS-HIE) System for Patient Monitoring, 2019 IEEE Symposium on Computers and Communications (ISCC), Barcelona, Spain, 2019, pp. 1-6, doi: 10.1109/ISCC47284.2019.8969718.

[5] Qing Zhang, Jian Gao, Qiqiang Qin, Chenyu Wang, and Keting Yin. FutureText: A Blockchain-based Contract Signing Prototype with Security and Convenience[C]. In Proceedings of the 3rd ACM International Symposium on Blockchain and Secure Critical Infrastructure (BSCI '21). Association for Computing Machinery, New York, NY, USA, 77-83.

[6] Zhang Jing. Discussion on the Innovation of Housing Provident Fund Management in the Era of Big Data [J]. Operators,2020,34(9):44-44.

[7] Li F. The role of data analysis of electronic archives in risk prevention of provident fund management [J]. Archives World,2018(8):58-59.

[8] See how the innovative application of "blockchain + housing provident fund" can beat the times, 2018. [Online]. Available: http://www.lianmenhu.com/blockchain-7633-1

[9] Leon, Cai. et al. Practice and Thinking of Alliance Blockchain. COMMUNICATIONS OF THE CCF, Vol 2, 2020, pp. 60-66

[10] Sun Zhixin, Zhang Xin, Xiang Feng, Chen Lu. Research progress of blockchain storage scalability [J]. Journal of Software,2021,32(01):1-20. 